American Journal of Infectious Diseases 1 (1): 55-60, 2005

ISSN 1553-6203

(C) 2005 Science Publications

\title{
Periodicity of the HIV/AIDS Epidemic in a Mathematical Model that Incorporates Complacency
}

\author{
Flugentius Baryarama, Livingstone S. Luboobi and Joseph Y.T. Mugisha \\ Department of Mathematics, Makerere University, Box 7062, Kampala, Uganda
}

\begin{abstract}
An HIV/AIDS model incorporating complacency for the adult population is formulated. Complacency is assumed a function of the number of AIDS cases in a community with an inverse relation. A method to find the equilibrium state of the model is given by proving a stated theorem. An example to illustrate the application of the theorem is also given. Model analysis and simulations show that complacency resulting from dependence of HIV transmission on the number of AIDS cases in a community leads to damped periodic oscillations in the number of infective with oscillations more marked at lower rates of progression to AIDS. The implications of these results to public health with respect to monitoring the HIV/AIDS epidemic and widespread use of antiretroviral (ARV) drugs is discussed.
\end{abstract}

Key words: HIV/AIDS, periodicity, mathematical model, complacency

\section{INTRODUCTION}

Complacency is used to mean to "relax" or "revert" to high risk sexual behaviours such as multiple sexual partners, sex with prostitutes and non-condom use once the HIV prevalence reduces to very low levels, with the number of AIDS cases becoming less in the community. Complacency is used in the context of a community that has registered significant decreases in HIV prevalence. This is the case for most Ugandan communities. Uganda has registered decreasing HIV prevalence since the early 1990s both nationally and based on community studies of selected high risk subpopulation ${ }^{[1-6]}$. To model complacency, it is assumed that behaviour change depends on the number of AIDS patients (HIV infected persons with fully blown AIDS symptoms) in the community. This dependence has been alluded to by a number of authors such as Leaman and Bhupal ${ }^{[7]}$ and Okware et al. ${ }^{[8]}$.

Recurrent behaviour is a key feature of most epidemics including nutrition related epidemics such as Kwashiorkor and Pellagra. A report on an investigation on the pellagra disease in Kulto, Angola showed annual epidemics of the disease with major outbreaks in June to September associated with the maize harvesting season ${ }^{[9]}$. Clearly the observed periodicity was due to seasonal variations. A number of diseases show such seasonal variations for a number of reasons including school calendars, weather conditions and behaviours in response to such seasonal events. Infectious diseases like measles, tuberculosis, typhus fever, influenza, chicken pox and several sexually transmitted diseases have been documented to show recurrent outbreaks that are less dependent on seasonal variations. It is unlikely that HIV/AIDS would exhibit seasonal variations either, though seasons do indeed impact on sexual behaviour and hence transmission of HIV.

Velasco-Hernandez et $a .^{[10]}$ on the effects of treatment and prevalence-dependent recruitment on the dynamics of fatal diseases, showed periodic solutions with a per- susceptible incidence rate of the form $\beta=\left(c \beta_{0} U+c^{\prime} \beta_{I} I\right) / N$ where $U, I$ are the untreated and treated infective and $N=U+I ; \beta_{0}, \beta_{I}$ are the per susceptible incidence among the untreated and treated infective respectively and $c, c^{\prime}$ are the respective contact rates. Hence treatment of AIDS patients could lead to periodicity of the HIV epidemic with resurgences in number of new HIV infections.

On a longer time scale, observed periodicity of epidemics has been attributed to changes in the population sizes of constituent epidemiological classes relative to each other as discussed by several authors ${ }^{[11-}$ ${ }^{14]}$. Such periodicity is imposed by the dynamics of the disease. In the case of HIV/AIDS these dynamics take various forms. First the subpopulations at various risks for HIV infection including age structure, second the stratification by stage of HIV infection and third the spatial distribution of communities and their interconnectedness. From this literature, it is clear that heterogeneity of the population is conducive for recurrent epidemics at longer time scales than those caused by seasonal variations.

The HIV/AIDS dynamics are known to give an SIR model. A general feature of SIR models, stochastic or deterministic, is that they give damped oscillations around a stable steady-state solution. In the case of HIV/AIDS, damped oscillations have been shown by Simwa and Pokhariyal ${ }^{[15]}$ using back calculation methods to derive incidence estimates from annual AIDS case reports for Kenya and Uganda.

Corresponding Author: Flugentius Baryarama, Department of Mathematics, Makerere University, Box 7062, Kampala, Uganda 
Their incidence curves show recurrent behavior with damped oscillations.

In this study, we show that complacency could lead to periodic behavior of the HIV/AIDS epidemic. This should be expected since complacency leads to a return to higher risk behaviors and hence to increased number of new HIV infections. It is assumed that reductions in the number of AIDS cases in a community lead to complacency resulting in the community reverting to high-risk sexual behaviors. The need to combat complacency in HIV prevention has already been voiced in developed countries like the USA and Britain with regard to extensive use of $\mathrm{ARVs}^{[7,16,17]}$. Ignoring safer sex messages when condoms are effective in preventing not only HIV but other sexually transmitted infections and unwanted pregnancies has been described as "misplaced" and the stigma attached to HIV/AIDS as "a great enemy"[7].

\section{MODEL PARAMETERS AND ASSUMPTIONS}

We formulate an HIV/AIDS model with complacency by considering the adult population in a one group, one stage model. At time $t$, there are $S(t)$ adult susceptibles, $I(t)$ infectives who are the infected and infectious individuals that have not yet developed AIDS symptoms and $A(t)$ AIDS cases who are infected and with AIDS symptoms. Susceptibles have sexual contacts at a rate $c$ with a probability of transmission at one sexual encounter denoted by $\beta$. A proportion of these sexual contacts are with infectives. We shall assume that this proportion is equal to the prevalence of infective in the population. The model upholds the common assumption of no sexual contacts with AIDS cases, though the role of sexual contacts with persons with AIDS symptoms may become important with advances in medical interventions. Sexual contacts within susceptible do not result in any transmission and thus, do not feature in the model. Also sexual contacts within infectives which gives rise to issues about the role of re-infection are ignored.

Denote the probability of transmission $\beta$ and the contact rate $c$ at the onset of the epidemic as $\beta_{0}$ and $c_{0}$, respectively. Assume that $\beta c$, at future points in time differs from $\beta_{0} c_{0}$ at the beginning of the epidemic and that there is an inverse relationship between $\beta c$ and the number of AIDS cases, i.e. $\beta c$, decreases with an increasing number of AIDS cases and increases with decreasing numbers of AIDS cases. The decrease in $\beta c$, with increases in number of AIDS cases is attributed to awareness and behaviour change. The increase in $\beta c$, with decreasing numbers of AIDS cases is attributed to complacency.

Our model allows for a generalized form of $\beta c$, which as per the above assumptions is a function of the number of AIDS cases denoted by $\eta(A)$ in the rest of this chapter. The objective is to investigate the implication of the dependence of the risk of transmission of HIV on the number of persons with AIDS symptoms in a community. The dependence of $\beta c$, on the number of AIDS cases has been alluded to by a number of researchers ${ }^{[7,18]}$.

\section{DERIVATION OF EQUATIONS OF THE MODEL}

The equations derived are for the adult population as shown in the description of each epidemiological class below.

Susceptibles, $S(t)$ : Consider a variable recruitment rate $\Lambda(\mathrm{t})$ to the susceptible population per unit time. Recruited individuals consist of maturing young persons joining the sexually active age group at a predetermined age. The recruitment term can be rewritten in terms of birth rates, maturation rates and rates of mother-to-child transmission with time lags ${ }^{[19]}$. Susceptibles are removed through infection or by natural death. We let $\mu$ be the natural death rate for the sexually active adults. The removal rate of susceptibles through infection is the number of new HIV infections per unit time. This rate is important in calculating HIV incidence which by definition is the number of new infected persons in a specified time period divided by the number of uninfected persons that were exposed for this same time period.

Number of new HIV infections: Let each susceptible have $c$ sexual contacts per unit time. Assume that a proportion $I / N$ of these contacts are with infectives and at each of these sexual contacts with infectives, a susceptible has a probability $\beta$, of getting infected. Let $\beta c$ be a function of the number of AIDS cases given by $\eta(A)$, then the total probability of one susceptible getting HIV infected from any of their sexual contacts per unit time is $\eta(A(t)) I / N$. This is the expression for the force of infection. The force of infection is the probability that a susceptible will get an HIV infection per unit time. Therefore in a population of $S$ susceptible, the number of new HIV infections per unit time is given by $\eta(A(t)) I S / N$.

Infectives, $\boldsymbol{I}(\boldsymbol{t})$ : Infectives are recruited through new HIV infections described above and removed through progression to AIDS at rate $v$ and through natural death at rate $\mu$. Hence $l / v$ is the duration spent in the infective stage and $1 / \mu$ is the life expectancy of the adult population. Both of these rates are assumed constant in the model.

AIDS cases, $\boldsymbol{A}(\boldsymbol{t})$ : AIDS cases are recruited through progression from the infective stage to the AIDS stage and removed through AIDS accelerated deaths at rate $\sigma+\mu$ where $1 / \sigma$ is the average duration spent in the AIDS stage if natural deaths are assumed constant in the model. However, allowing for variability in $\sigma$ could 
be necessary given the advances in medical interventions and in changes in medical seeking behaviours for persons living with HIV/AIDS.

\section{MODEL EQUATIONS}

From the descriptions and assumptions on the dynamics of the epidemic made above, the following are the model equations.

$$
\left.\begin{array}{l}
\frac{\mathrm{d} S}{\mathrm{dt}}=\Lambda(\mathrm{t})-\eta(\mathrm{A}) \frac{\mathrm{SI}}{\mathrm{N}}-\mu \mathrm{S} \\
\frac{\mathrm{dI}}{\mathrm{dt}}=\eta(\mathrm{A}) \frac{\mathrm{SI}}{\mathrm{N}}-(\nu+\mu) \mathrm{I} \\
\frac{\mathrm{dA}}{\mathrm{dt}}=\nu \mathrm{I}-(\sigma+\mu) \mathrm{A}
\end{array}\right\}
$$

\section{ANALYSIS OF THE MODEL}

Let us focus on the equilibrium state of the system and its stability.

Equilibrium in the $(I, \boldsymbol{A})$ plane: Suppose that at the equilibrium state in $(I, A)$, the number of susceptibles $S$ continues to increase and hence both $S$ and $N$ vary. Setting the derivative in the third equation in equations (1) to zero, we get

$$
A^{*}=\frac{v}{\sigma+\mu} I^{*} \text { or } I^{*}=\frac{\sigma+\mu}{v} A^{*}
$$

Similarly, from the second equation, we get

$$
\frac{S}{N}-\frac{(v+\mu)}{\eta\left(A^{*}\right)}=0, \quad \text { or } \quad \frac{S}{N}=\frac{(\nu+\mu)}{\eta\left(A^{*}\right)}
$$

But

$$
\frac{S}{N}+\frac{I^{*}}{N}=1
$$

Hence,

$$
\frac{v+\mu}{\eta\left(A^{*}\right)}+\frac{(\sigma+\mu)}{v} \frac{A^{*}}{N}=1
$$

We state the following theorem.

Theorem: Suppose for equations (1), $S(t) \rightarrow \infty$ as $t \rightarrow \infty$. Further suppose that $\eta(A)$ has an inverse, $\eta^{-1}$ on $(0,1)$. Then there exists $t_{A}>0$ and $\varepsilon_{A}>0$ such that for all $t>t_{A},\left|A(t)-A\left(t_{A}\right)\right|<\varepsilon_{A}$ and $\left|I(t)-I\left(t_{A}\right)\right|<\varepsilon_{A}$. Moreover $S(t) \rightarrow \infty$ as $\mathrm{t} \rightarrow \mathrm{t}_{\mathrm{A}}$.

Proof: Suppose $S(t) \rightarrow \infty$ as $t \rightarrow \infty$, then from (3) $S / N \rightarrow 1$ (and $I / N \rightarrow 0, A / N \rightarrow 0$ ). From (2), it follows that $\eta(A(t)) \rightarrow v+\mu=\eta\left(A^{*}\right)$ as $\mathrm{t} \rightarrow \infty$. Hence $A^{*}=\eta^{-1}(v+\mu)$ and for $t_{A}$ sufficiently large, there exists an $\varepsilon_{l}>0$ such that for all $t>t_{A},\left|A(t)-A\left(t_{A}\right)\right|<\varepsilon_{l}$.
But

$I^{*}=\frac{\sigma+\mu}{v} A^{*}=\frac{\sigma+\mu}{v} \eta^{-1}(v+\mu)$

Hence, for sufficiently large $t_{A}$, for all $t>t_{A}$,

$\left|I(t)-I\left(t_{A}\right)\right|<\frac{\sigma+\mu}{v} \varepsilon_{1}=\varepsilon_{2}$

Choosing $\varepsilon_{A}=\varepsilon_{2}$ since $\varepsilon_{2}>\varepsilon_{2}$ ends the proof for the first part.

From (2),

$\frac{S}{S+I^{*}}-\frac{v+\mu}{\eta\left(A^{*}\right)}=0$, or $S=\left(S+I^{*}\right) \frac{v+\mu}{\eta\left(A^{*}\right)}$

Simplifying gives

$S=\frac{(v+\mu) I^{*}}{\eta\left(A^{*}\right)-(v+\mu)}$

Since $\eta(A(t)) \rightarrow(v+\mu)$ as $t \rightarrow t_{A}$ then $S \rightarrow \infty$ as $t \rightarrow t_{A}$. Hence the behaviour of $S(t)$, for $t>t_{A}$ remains of little consequence to $A^{*}$ and $I^{*}$ since $S>S\left(t_{A}\right)>>I^{*}>A^{*}$. Hence, the end of proof for the second part of the theorem.

It follows from the above theorem that explicit expressions for the equilibrium

point $E^{1}$ in $(A, I)$ are

$A^{*}=\eta^{-1}(v+\mu)$ and

$I^{*}=\frac{\sigma+\mu}{v} \eta^{-1}(v+\mu)$

An Example of Linear $\eta(A)$ : Let $\eta(A)=\left(\eta_{0}-\eta_{1} A\right)$.

Then $\quad \eta^{-1}=\frac{\eta_{0}}{\eta_{1}}-\frac{\eta}{\eta_{1}} \quad$ From the theorem, $A^{*}=\eta^{-1}(v+\mu)=\frac{\eta_{0}}{\eta_{1}}-\frac{v+\mu}{\eta_{1}}$ and $I^{*}=\frac{\sigma+\mu}{v}\left(\frac{\eta_{0}}{\eta_{1}}-\frac{v+\mu}{\eta_{1}}\right)$

This result can alternatively be derived by setting $d I / d t=0$ and $d A / d t=0$ simultaneously with the assumption that $S / N \rightarrow 1$ as $t \rightarrow \infty$ as shown below:

Rewrite equations for $I$ and $A$ as

$\frac{d I}{d t}=\eta(A) \frac{S I}{N}-(v+\mu) I$

$\frac{d A}{d t}=v I-(\sigma+\mu) A$

The $A$-isocline is $I=(\sigma+\mu) A / v$ and the $I$-isocline is $\eta(A) S /(S+I)=v+\mu$. Hence, the $I$-isocline is in general a curve while the $A$-isocline is a straight line through the origin. Thus, $\eta(A) S=(v+\mu)(S+I)$, or

$I=S \frac{\eta(A)-(v+\mu)}{v+\mu}$ 
Solving the isocline equations simultaneously for $\eta(A)=\eta_{0}-\eta_{1} A$ to get their point of intersection, we have

$$
\frac{(\sigma+\mu) A}{v}=S \frac{\left(\eta_{0}-\eta_{1} A\right)-(v+\mu)}{v+\mu}
$$

Simplfying, gives

$$
A=\frac{S \frac{\eta_{0}-(\nu+\mu)}{\nu+\mu}}{\frac{\sigma+\mu}{\nu}+\frac{\eta_{1} S}{v+\mu}}
$$

Dividing top and bottom by $S$ and taking the limit as $S \rightarrow \infty$, gives $A^{*}=\eta_{0} / \eta_{1}-(\nu+\mu) / \eta_{1}$

Hence,

$$
I^{*}=\frac{\sigma+\mu}{v} A^{*}=\frac{\sigma+\mu}{v}\left[\frac{\eta_{0}}{\eta_{1}}-\frac{v+\mu}{\eta_{1}}\right]
$$

Thus the $\left(A^{*}, I^{*}\right)$ obtained by this direct method is the same as solutions got by using the theorem.

The stability of the equilibrium point $E^{1}$ : A stability analysis shows that the resulting trajectory in the $(A-I)$ plane is a a clockwise steady spiral toward the equilibrium point $\left(A^{*}, I^{*}\right)$. The spiral for $\eta(A)=0.433-0.001 A$ is shown in Fig. 1 for linear $\eta(A)$; and its time evolution is shown in Fig. 2. Simulations with non-linear forms of $\eta(A)$, (not shown), showed similar periodic behaviour.

Variability of oscillations with removal rate of AIDS cases from the community: The effect of varying the rate of removal of AIDS cases on the oscillations in number of HIV infectives and number of AIDS cases in a community was investigated by studying the $I(t)$ time trends and the $I(t)-A(t)$ phase portraits for varying values of the mean life time spent in the AIDS stage at 2 years $(\sigma=0.5), 4$ years $(\sigma=0.25)$ and 8 years ( $\sigma=0.125$ ). From Fig. 3, oscillations are rare when AIDS cases stay for short time periods in the community as seen for 2 years, $(\sigma=0.5)$. However, when AIDS symptomatic cases stay longer in the community the number of infectives become lower and there is an increased tendency for damped periodic behaviour as is shown for 4 years $(\sigma=0.25)$ and 8 years $(\sigma=0.125)$. The simulations show that although the endemic number of infectives reduces with decreasing rates of removal of AIDS cases from 1200 for $\sigma=0.5$ to 330 for $\sigma=0.125$, the endemic number of AIDS cases remain insensitive to changes in rate of removal of AIDS cases at about 280 AIDS cases. a) Number of AIDS cases (x) versus number of infectives (y) phase portrait

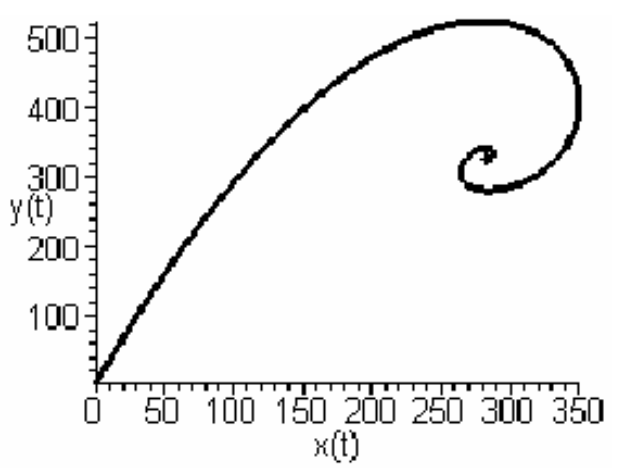

Fig. 1: The $A-I$ phase portrait for $\eta(A)=0.433-0.001 A$ and $\nu=0.125, \mu=.02$

b) Number of infectives (y) over time in years

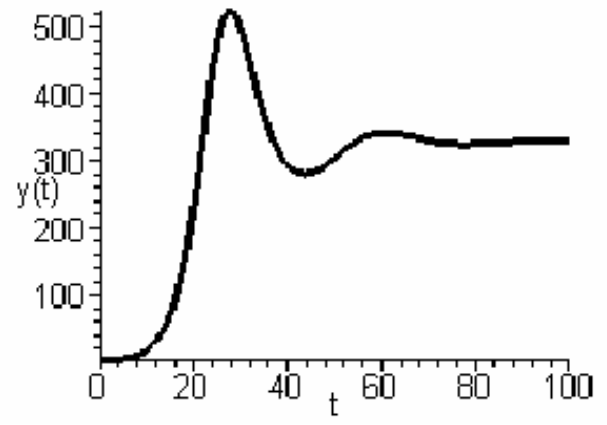

Fig. 2: The $I$-time trends for $\eta(A)=0.433-0.001 A$ and $\nu=0.125, \mu=.02$

The effect of varying the rate of progression to fully blown AIDS was also investigated (data not shown). The mean time from infection to development of AIDS symptoms was varied from 8 years $v=0.125$ ) to 16 years $(\nu=0.063)$.

Spending longer time before showing HIV symptoms leads to higher number of infectives as well as higher numbers of AIDS cases in the community. This also leads to an overall higher endemic number of infectives and AIDS cases. However rate of progression did not independently affect the periodic behaviour of the model.

Use of exponential dependence or lower bounded dependence lowered the number of infectives and AIDS cases as well as the equilibrium levels attained (data not shown). However use of these forms of $\eta(A)$ did not change the periodic behaviour of the model. 



Mant

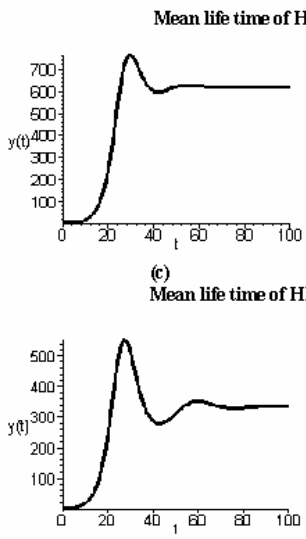

(e)

Mean life time of HW/AIDS cases $=8$ years (e and $f$ )

Fig. 3: The $I$-time trends and $I-A$ phase portraits for $\eta(A)=0.433-0.001 A$ and $\nu=0.125, \mu=.02$ and varying removal rate of AIDS cases

\section{DISCUSSION}

The model formulated shows that complacency could lead to damped oscillations in number of persons infected with HIV. This however may not translate into oscillations in HIV prevalence since the susceptible population size is assumed to keep increasing at low prevalence levels and the period of the oscillations is of the order of several years. Instead, the HIV prevalence may exhibit a sustained decline or may become stable. Assuming that the first decade from introduction of an HIV infected individual corresponds to very low infection rates so that the HIV epidemic begins at the beginning of the second decade, this complacency model suggests that the second wave of the epidemic begins 30 years from the beginning of the epidemic. The model shows a tendency for the epidemic to stabilise at higher numbers of infectives and AIDS cases than the minimum numbers attained during the first decline of the epidemic.

A method for finding the equilibrium point given the transmission term $\eta(A)$ was provided through a proof to a theorem. The equilibrium point in terms of $\eta^{-1}$ is $\left(\eta^{-1}(v+\mu), \frac{\sigma+\mu}{v} \eta^{-1}(v+\mu)\right)$ in the $(A, I)$ plane. Hence the equilibrium point is readily found for any invertible $\eta(A)$ as we showed for linear $\eta(A)$. A stability analysis showed that $\left(A^{*}, I^{*}\right)$ is approached through a clockwise steady spiral. This was verified with numerical solutions. The numerical solutions further showed that the oscillations are more marked for low rates of progression to AIDS. This supports findings, fears and debates that extensive use of ARVs could result in resurgent HIV epidemics. The model however shows that prolonging survival of AIDS cases lowers the endemic equilibrium level and is hence desirable.

The main limitation to our analysis may be that we did not account for the effect of behaviour change arising from interventions that are independent of the number of AIDS cases in the community. Though a detailed analysis of this was not undertaken, simple assumptions of behaviour change suggest more marked damping and less marked oscillations may result from such an allowance. Secondary, the possible effects of extensive use of ARVs in terms of method of distributing drugs through public or private health institution or a combination of both could determine whether patients on ARVs revert back to the infective class. This together with reduced infectiousness due to lower viral loads for those on treatment was not accounted for.

Despite the limitations, there are several implications of these findings to public health. One, the endemic equilibrium should be brought as low as possible especially during the first wave of the epidemic. This model suggests that this can be achieved by prolonging the life time of the AIDS patients for as long as possible. Two, HIV prevalence at low prevalence levels become less sensitive to changes in the dynamics of HIV epidemic because it is overpowered by demographic changes especially the recruitment of susceptibles. At low prevalence levels, there is hence need to track trends in number of persons infected with HIV than tracking HIV prevalence.

\section{ACKNOWLEDGEMENT}

The authors are grateful to NUFU for financial support during preparation and publication of this study and to the anonymous referee for the helpful comments on the original manuscript.

\section{REFERENCES}

1. Baryarama, F., R.E. Bunnell and R.L. Ransom, 2004. Using HIV voluntary counselling and testing data for monitoring the Uganda HIV epidemic, 1992- 2000. J. Acquir. Immun. Defic. Syndr., 37: 1180-1186.

2. Fabiani, M., C. Ble, C. Grivel and M. Lukwiya, 1998. 1989-1986 HIV-1 prevalence trends among different risk groups in Gulu District, North Uganda. J. Acquir. Immun. Defic. Syndr., 18: 781.

3. Kamali, A., L.M. Carpenter, J.A.G. Whitworth and G. Pool, 2000. Seven-year trends in HIV-1 infection rates and changes in sexual behaviour among adults in rural Uganda. AIDS, 14: 427-434. 
4. Mbulaiteye, S.M., C. Mahe, J.A.G. Whitworth, A. Ruberantwari and J.S. Nakiyingi, 2002. Declining HIV-1 incidence and associated prevalence over a 10 -year period in a rural population cohort in south-west Uganda. Lancet, 360: 41-46.

5. UNAIDS/Uganda, 2000. Uganda Epidemiological Fact Sheet on HIV/AIDS and Sexually Transmitted Diseases, Geniva: UNAIDS; DHS 1995, 2000/2001.

6. USAID, 2003. Uganda country profile. http://www.usaid.gov/pophealth/aids/Countries/afri ca/uganda.html.

7. Leaman, J. and N. Bhupal, 2000. New figures suggest worsening public complacency of HIV threat in Britain. http://www.mori.com/polls/2000/nat.shml.

8. Okware, S., A. Opio, J. Musinguzi and P. Waibale, 2001. Fighting HIV/AIDS: is success possible? Bulletin of WHO, 79: 1113-1120.

9. Golden, M., 2002. A report on the investigation into recurrent epidemics of pellagra in Kulto, Angola. www.fantaproject.org.

10. Velasco-Hernandez, J.X., F. Brauer and C. Castillo-Chavez, 1996. Effects of treatment and prevalence-dependent recruitment on the dynamics of a fatal disease. IMA J. Math. Appl. Med. Biol., 13: $175-192$.
11. Anderson, R.M. and A.R. McLean, 1988. Possible demographic consequences of AIDS in developing countries. Nature, 332: 228-234.

12. Gao, L.Q., J. Mena-Lorca and H.W. Hethcote, 1995. Four SEI epidemic models with periodicity and separatrices. J. Math. Biol., 128: 157-184.

13. Grenfell, B., B.M. Bolker and M. Kleczkowski, 1994. Seasonality and extinction in chaotic metapopulations. I.C.T.P., Trieste, Italy.

14. Kuznetsov, Y.A. and C. Piccardi, 1994. Bifurcation analysis of periodic SEIR and SIR epidemic models. J. Math. Biol., 32: 109-121.

15. Simwa, R.O. and G.P. Pokhariyal, 2003. On empirical modelling of HIV/AIDS pandemic with application to East Africa. AJST, 4: 104-109.

16. BBC News Online, 2001. Warning over AIDS complacency. http://news.bbc.co.uk/1/hi/health/1406793.stm

17. Centers for Disease Control and Prevetion (CDC), 1998. Combating complacency in HIV Prevention. June 1998 Report.

18. Peterman, T.A., A.A. Zaidi and M. Wroten, 1995. Decreasing prevalence hides a high HIV incidence: Miami. AIDS, 8: 811-817.

19. Luboobi, L.S., 1994. A three-age groups model for the HIV/AIDS epidemic in Uganda. Math. Comp. Model., 19: 99-105. 